\title{
Logic-Based Modeling in Systems Biology
}

\author{
Alexander Bockmayr \\ DFG-Research Center Matheon, FB Mathematik und Informatik, \\ Freie Universität Berlin, Arnimallee 6, 14195 Berlin, Germany \\ Alexander.Bockmayr@fu-berlin.de
}

\begin{abstract}
Systems biology is a new interdisciplinary research field that has received considerable attention in recent years. While traditional molecular biology studies the various components of a biological system (e.g. genes, RNAs, proteins) in isolation, systems biology aims to understand how these components interact in order to perform complex biological functions.

A variety of mathematical and computational methods is currently being used to model and analyze biological systems, ranging from continuous, stochastic, and discrete to various hybrid approaches.

In this talk, we focus on logic-based methods for systems biology, which arise at two distinct levels. On the one hand, Boolean or multi-valued logics provide a natural way to represent the structure of a regulatory biological network, which is given by positive and negative interactions (i.e., activation and inhibition) between its different components. On the other hand, temporal logics (e.g. CTL or LTL) may be used to reason about the dynamics of a biological system, represented by a state transition graph or Kripke model.
\end{abstract}

